\title{
Beacons in the sky: Classical novae vs. X-ray bursts
}

\author{
J. José ${ }^{1, a}$ and M. Hernanz ${ }^{2}$ \\ 1 Institut d'Estudis Espacials de Catalunya (IEEC-UPC) and Departament de Física i Enginyeria Nuclear, Universitat \\ Politècnica de Catalunya, Barcelona, Spain, \\ 2 Institut d'Estudis Espacials de Catalunya (IEEC-CSIC) and Institut de Ciències de l'Espai (CSIC), Bellaterra, Spain
}

Received: 22 June 2005 /

Published online: 24 February 2006 - C Società Italiana di Fisica / Springer-Verlag 2006

\begin{abstract}
Thermonuclear runaways are at the origin of some of the most energetic and frequent stellar cataclysmic events. In this review talk, we outline our understanding of the mechanisms leading to classical nova explosions and X-ray bursts, together with their associated nucleosynthesis. In particular, we focus on the interplay between nova outbursts and the Galactic chemical abundances (where ${ }^{13} \mathrm{C},{ }^{15} \mathrm{~N}$, and ${ }^{17} \mathrm{O}$ constitute the likely imprints of many nova outbursts during the overall $10 \mathrm{Gyr}$ of Galactic history), the synthesis of radioactive nuclei of interest for gamma-ray astronomy $\left({ }^{7} \mathrm{Be}-{ }^{7} \mathrm{Li},{ }^{22} \mathrm{Na}\right.$, or $\left.{ }^{26} \mathrm{Al}\right)$, the endpoint of nova nucleosynthesis, based on theoretical and observational grounds, and the recent discovery of presolar meteoritic grains, both in the Murchison and Acfer 094 meteorites, likely condensed in nova shells. Recent progress in the modeling of X-ray bursts as well as an insight into the input nuclear physics requests, for both novae and X-ray bursts, will also be presented.
\end{abstract}

PACS. 26.50.+x Nuclear physics aspects of novae, supernovae, and other explosive environments 26.30. $+\mathrm{k}$ Nucleosynthesis in novae, supernovae and other explosive environments -95.85 .Pw $\gamma$-ray 97.80.Gm Cataclysmic binaries (novae, dwarf novae, recurrent novae, and nova-like objects); symbiotic stars

\section{Introduction}

Classical novae and type Ia (or thermonuclear) supernovae have been extensively observed during the past two millennia. They constitute dramatic stellar explosions occurring in interacting binary systems, where a compact white dwarf (the stellar remnant of a Main Sequence star with a mass below $\sim 10 \mathrm{M}_{\odot}$; white dwarfs have planetary dimensions and masses typically in the range $0.6-1.4 \mathrm{M}_{\odot}$ ) accretes material from a low-mass Main Sequence companion. In contrast, X-ray bursts have been discovered much recently, since a major fraction of their energy output is emitted in X-rays, and hence, detection requires the use of space observatories. About 65 Galactic low-mass $\mathrm{X}$-ray binaries that exhibit such bursting behavior have been found since the independent discovery by Grindlay et al. (1976) [1] and Belian et al. (1976) [2]. In these systems, the explosion takes place in an even more compact stellar remnant: a neutron star (with a mass about 1-2 $\mathrm{M}_{\odot}$, and a very small diameter, 20 to $30 \mathrm{~km}$ only; neutron stars formation accompanies some type II supernova explosions for stars more massive than $\sim 10 \mathrm{M}_{\odot}$, but can be formed also in other processes such as the so-called "accretion induced collapse" of a white dwarf; see Swank et

\footnotetext{
${ }^{a}$ e-mail: jjose@ieec.fcr.es
}

al. (1978) [3] for an account of the identification of a neutron star as the central emitting source in the bursting system $3 \mathrm{U} 0614+09)$. In all these cataclysmic systems, mass transfer episodes, caused by Roche lobe overflows of the Main Sequence companion, proceed through the inner Lagrangian point of the system, forming an accretion disk around the compact star. A fraction of the material contained in the accretion disk ultimately falls on top of the white dwarf (as a result of angular momentum losses driven by dissipative forces in the disk), where it is gradually compressed up to degenerate conditions, thus leading to a thermonuclear runaway (TNR). The estimated number of nova events in our Galaxy is about $30 \pm 10 \mathrm{yr}^{-1}$ (Shafter, 2002, [4]). Contrary to type Ia supernovae, for which the white dwarf is fully disrupted by the strength of the explosion, classical novae are expected to recur within a timescale of the order of $10^{4}-10^{5} \mathrm{yr}$ (Warner, 1995, [5]); in contrast, X-Ray bursts recur on timescales from hours to days (see Strohmayer and Bildsten, 2006, [6] for a recent review). These stellar explosions are characterized by a remarkable energy output, of the order of $10^{39}$ ergs (Xray bursts), $10^{45}$ ergs (classical novae), and $10^{51}$ ergs (supernovae). A major difference between these cataclysmic events is the amount of mass ejected as well as the mean ejection velocities: whereas in a type Ia supernova, the whole star (cf., $1.4 \mathrm{M}_{\odot}$ ) is ejected at $>10^{4} \mathrm{~km} \mathrm{~s}^{-1}$, the 
explosion in both classical novae and X-ray bursts is restricted to the accreted envelope. Hence, $10^{-4}-10^{-5} \mathrm{M}_{\odot}$ are typically ejected during nova outbursts, at mean ejection velocities ranging from $10^{2}-10^{3} \mathrm{~km} \mathrm{~s}^{-1}$. In contrast, it is likely that in a typical X-ray burst no mass ejection takes place, because of the extreme escape velocities from a neutron star (the energy required to escape from the strong gravitational field of a neutron star of mass $M$ and radius $R$ is $G M m_{p} / R \sim 200 \mathrm{MeV} /$ nucleon, whereas the nuclear energy released from fusion of solar-like matter into Fe-group elements is just $\sim 5 \mathrm{MeV} /$ nucleon).

\section{Classical nova explosions}

The canonical scenario of classical novae consists of an initially low-luminosity white dwarf accreting H-rich matter from a low-mass Main Sequence companion, at an accretion rate low enough to allow the building-up of an envelope where the TNR takes place. Accretion compresses matter to large densities, such that material becomes degenerate. Once H-ignition conditions are reached, a thermonuclear runaway takes place, since degenerate matter cannot expand when heated. The large energetic output produced by nuclear reactions cannot be only evacuated by radiation, and hence convection sets in and transports the $\beta^{+}$-unstable nuclei to the outer cooler regions where their decay provokes the nova outburst: the sudden release of energy from those $\beta^{+}$-decays increases the temperature and the entropy of the material; as a result, the envelope degeneracy is lifted and expansion sets in, paving the road to the ejection stage. Therefore, the TNR is halted by the envelope expansion rather than by fuel consumption. Another important effect of convection is that it transports fresh unburned material to the burning shell, and hence, non-equilibrium burning occurs and the resulting nucleosynthesis will be far from that of hydrostatic hydrogen burning. It is important to stress that significant amounts of CNO nuclei are required to let the ignition process really progress into a thermonuclear runaway and indeed to get an (energetic enough) explosion, and also to account for the inferred abundances from nova shells. Mixing episodes between the solar-like accreted envelope matter and the outer layers of the underlying white dwarf core have been invoked to account for the source of those CNO nuclei.

Nova explosions can occur in two types of white dwarfs: carbon-oxygen $(\mathrm{CO})$ and oxygen-neon $(\mathrm{ONe})$. The first one correspond to the end-product of a star with a mass below $\sim 10 \mathrm{M}_{\odot}$, which undergoes two evolutionary stages, central hydrogen burning and central helium burning, leaving a carbon and oxygen-rich object $(\mathrm{CO})$ supported by electron pressure (in contrast, neutron stars balance the strong gravitational force by means of neutron pressure). More massive progenitors undergo an additional stage, that is non-degenerate carbon ignition, leading to the formation of an oxygen and neon-rich core $(\mathrm{ONe})$, with traces of magnesium and sodium. The cut-off mass, that somewhat distinguishes both white dwarf subclasses, is about $1.1 \mathrm{M}_{\odot}$. It is important to stress that historically, these cores were thought to be made of oxygen, neon and magnesium (the so-called $\mathrm{ONeMg}$ white dwarfs) on the basis of parametrized calculations of hydrostatic carbon burning (Arnett and Truran, 1969, [7]), but more recent, self-consistent models of sAGB (super-asymptotic giant branch) stars, following the thermally pulsing phases, have shown that the amount of ${ }^{24} \mathrm{Mg}$ in these cores is much smaller than expected (i.e., smaller than ${ }^{23} \mathrm{Na}$; see Domí nguez et al., 1993, [8]; Ritossa et al., 1996, [9]). It is also worth noting that these ONe white dwarfs have thick $\mathrm{CO}$ buffers on top of their very cores. Explosions on top of these objects (before a significant number of outbursts may erode the buffer) may be misclassified as non-neon (CO) novae, since their spectra will not show evidence for strong neon lines. These and other weird nucleosynthetic patterns have been predicted to accompany these explosions (see José et al., 2003, [10] for details).

It is important to stress that the injection of "seed" nuclei in the Ne-Si mass region, resulting from mixing with an $\mathrm{ONe}$ white dwarf, withdraws the main argument in support of CNO-breakout in classical novae. Indeed, recent estimates of several CNO-breakout reactions (see, for instance, Davids et al., 2003, [11] for ${ }^{15} \mathrm{O}(\alpha, \gamma)$ ), demonstrate that at the typical temperatures attained during nova outbursts the CNO cycle does not break out (contrary to what happens for X-ray bursts, for which peak temperatures are almost an order of magnitude higher than in classical nova outbursts).

In nova outbursts, the main nuclear activity is associated with $(\mathrm{p}, \gamma)$ and $(\mathrm{p}, \alpha)$ reactions, running between the line of stable nuclei and the proton drip line. Some of these rates have been measured at the right energy range in the laboratory since at the typical temperatures achieved during nova outbursts, measurements of nuclear cross sections in the region of the Gamow peak are, except for a few exceptions, feasible. In this sense, classical nova are unique stellar explosions, and represent the only explosive site for which the nuclear physics input is (or will be in the near future) primarily based on experimental information (see José et al., 2006, [12]).

\subsection{The role of nova outbursts in the galactic alchemy}

As discussed above, the moderately high peak temperatures achieved during nova explosions, $T_{\text {peak }} \sim(2-3) \times$ $10^{8} \mathrm{~K}$, suggest that abundance levels of the intermediatemass elements in the ejecta must be significantly enhanced, as confirmed by spectroscopic determinations in well-observed nova shells. This raises the issue of the potential contribution of novae to the Galactic abundances, which can be roughly estimated as the product of the Galactic nova rate $\left(\sim 30\right.$ events $\left.\mathrm{yr}^{-1}\right)$, the average ejected mass per nova outburst $\left(\sim 2 \times 10^{-5} \mathrm{M}_{\odot}\right)$, and the Galaxy's lifetime ( 10 Gyr). This order of magnitude estimate points out that novae scarcely contribute to the Galaxy's overall metallicity (as compared with other major sources, such as supernova explosions or AGB stars), but nevertheless they can substantially contribute to the synthesis of some largely overproduced species. Indeed, studies suggest that classical novae are likely sites 
for the synthesis of most of the Galactic ${ }^{13} \mathrm{C},{ }^{15} \mathrm{~N}$, and ${ }^{17} \mathrm{O}$, whereas they can partially contribute to the Galactic abundances of other species with $A<40$, such as ${ }^{7} \mathrm{Li},{ }^{19} \mathrm{~F}$, or ${ }^{26} \mathrm{Al}$ (Starrfield et al., 1998, [13]; José and Hernanz, $1998,[14])$. Because of the lower peak temperatures achieved in $\mathrm{CO}$ models (together with the lack of significant amounts of "seed" nuclei in the NeNa-MgAl region), the main nuclear activity in $\mathrm{CO}$ novae does not extend much beyond oxygen. In contrast, ONe models show a much larger nuclear activity, extending up to silicon (for $1.15 \mathrm{M}_{\odot} \mathrm{ONe}$ ) or argon (for $1.35 \mathrm{M}_{\odot} \mathrm{ONe}$ ). Hence, the presence of significantly large amounts of intermediatemass nuclei in the spectra, such as phosphorus, sulfur, chlorine or argon, may reveal the presence of an underlying massive ONe white dwarf. Another trend derived from the analysis of the nucleosynthesis accompanying nova outbursts is the fact that the $\mathrm{O} / \mathrm{N}$ and $\mathrm{C} / \mathrm{N}$ ratios decrease as the mass of the white dwarf (and hence, the peak temperature attained during the explosion) increases.

\subsection{The abundance pattern of nova shells}

In order to constraint models, several works have focused on a direct comparison of the atomic abundances inferred from observations of the ejecta with the theoretical nucleosynthetic output (see José and Hernanz, 1998, [14]; Starrfield et al., 1998, [13]; Kovetz and Prialnik, 1997, [15], and references therein; see also Yaron et al., 2005, [16]). This comparison itself is not very restrictive since observations provide only information on elemental abundances (i.e., for $\mathrm{O}$ we get the total contribution from ${ }^{16} \mathrm{O},{ }^{17} \mathrm{O}$, and $\left.{ }^{18} \mathrm{O}\right)$. Indeed, these atomic abundances are not absolute but relative to one element (often, hydrogen), and they cannot be directly inferred from observations: their determination involves modeling through photoionization codes or other numerical techniques. Nevertheless, and despite of the problems associated with the modeling of the explosion (Starrfield, 2002, [17]), there is, in general, good agreement between theory and observations as regards nucleosynthesis (i.e., including atomic abundances - H, He, $\mathrm{C}, \mathrm{O}, \mathrm{Ne}, \mathrm{Na}-\mathrm{Fe}-$, and a plausible endpoint for nova nucleosynthesis). In some cases, such as for PW Vul 1984, the agreement between observations and theoretical predictions (see José and Hernanz, 1998, [14], Table 5, for details) is quite remarkable. The reader is referred to Gehrz et al. (1998) [18] for an extended list of abundance determinations in the ejecta from novae.

Since the nuclear path is very sensitive to details of the evolution (chemical composition, extend of convective mixing, thermal history of the envelope...), the agreement between the inferred abundances and the theoretical yields suggests that the overall picture, in the framework of the thermonuclear runaway model, is reasonably well understood.

\subsection{Presolar grains}

Infrared (Evans, 1990, [19]; Gehrz et al., 1998, [18]) and ultraviolet observations of the temporal evolution of nova light curves suggest that novae form grains in the expanding nova shells. Both $\mathrm{CO}$ and ONe novae behave similarly in the infrared right after the outburst. However, as the ejected envelope expands and becomes optically thin, such behavior dramatically changes: CO novae are typically followed by a phase of dust formation corresponding to a decline in visual light, together with a simultaneous rise in the infrared emission (Rawlings and Evans, 2002, [20]; Gehrz, 2002, [21]). In contrast, it has been argued that ONe novae (that involve more massive white dwarfs than CO novae) are not so prolific producers of dust as a result of the lower mass, high-velocity ejecta, where the typical densities can be low enough to enable the condensation of appreciable amounts of dust. Indeed, infrared measurements in a number of recent novae have revealed the presence of C-rich dust (Novae Aql 1995, V838 Her 1991, PW Vul 1984, ...), SiC (Novae Aql 1982, V842 Cen 1986, ...), hydrocarbons (Novae V842 Cen 1986, V705 Cas 1993, ...), or SiO2 (Novae V1370 Aql 1982, V705 Cas 1993). Remarkable examples, such as nova QV Vul 1987, exhibited simultaneous formation of all those types of dust (see Gehrz et al., 1998, [18] for details on dust forming novae).

Since the first studies of dust formation in classical novae (Clayton and Hoyle, 1976, [22]), the identification of presolar nova grains in the laboratory (isolated from meteorites), presumably condensed in the shells ejected during the explosion, relied only on low ${ }^{20} \mathrm{Ne} /{ }^{22} \mathrm{Ne}$ ratios (with ${ }^{22} \mathrm{Ne}$ attributed to ${ }^{22} \mathrm{Na}$ in situ decay, rather than trapped ${ }^{22} \mathrm{Ne}$, since noble gases do not condense into grains). But quite recently, five silicon carbide and two graphite grains (isolated from both the Murchison and Acfer 094 meteorites) that exhibit isotopic signatures characteristic of nova nucleosynthesis, have been identified (Amari et al., 2001, [23]; Amari, 2002, [24]). These grains are characterized by low ${ }^{12} \mathrm{C} /{ }^{13} \mathrm{C}$ and ${ }^{14} \mathrm{~N} /{ }^{15} \mathrm{~N}$ ratios, ${ }^{30} \mathrm{Si}$ excesses and close-to or slightly lower-than-solar ${ }^{29} \mathrm{Si} /{ }^{28} \mathrm{Si}$ ratios, high ${ }^{26} \mathrm{Al} /{ }^{27} \mathrm{Al}$ ratios (determined only for two grains) and low ${ }^{20} \mathrm{Ne} /{ }^{22} \mathrm{Ne}$ ratios (only measured in the graphite grain KFB1a-161). This discovery provides valuable constraints for nova nucleosynthesis models (in contrast to observations of nova shells, for which only atomic abundances are inferred). Theoretical efforts to predict the expected imprints of nova outbursts on presolar grains have been conducted by several authors (Starrfield et al., 1997, [25]; José et al., 2003 [26]; 2004 [27]), including preliminary work on equilibrium condensation sequences in the ejected shells (José et al., 2004, [27]). These studies suggest that classical novae may contribute to the known presolar corundum $\left(\mathrm{Al}_{2} \mathrm{O}_{3}\right)$, spinel $\left(\mathrm{MgAl}_{2} \mathrm{O}_{4}\right)$, enstatite $\left(\mathrm{MgSiO}_{3}\right)$, silicon carbide $(\mathrm{SiC})$ and silicon nitride $\left(\mathrm{Si}_{3} \mathrm{~N}_{4}\right)$ grain populations. Aspects, such as the conditions that characterize grain formation in novae deserve more attention (see Shore and Gehrz, 2004, [28] for an insight into dynamic grain formation driven by photo-ionization).

\subsection{Nova radioactivities}

Among the isotopes synthesized during classical nova outbursts, several radioactive species have deserved special 
attention. Short-lived nuclei, such as ${ }^{14,15} \mathrm{O}$ and ${ }^{17} \mathrm{~F}$ (and to some extent ${ }^{13} \mathrm{~N}$ ) have been identified as the key isotopes that power the expansion and further ejection of the envelope during nova outbursts through a sudden release of energy, a few minutes after peak temperature (Starrfield et al., 1972, [29]). Other isotopes have been extensively investigated in connection with the predicted $\gamma$-ray output from novae (Clayton and Hoyle, 1974, [30]; Clayton, 1981, [31]; Leising and Clayton, 1987, [32]). Hence, ${ }^{13} \mathrm{~N}$ and ${ }^{18} \mathrm{~F}$ are responsible for the predicted prompt $\gamma-$ ray emission (Hernanz et al., 1999, [33]) at and below $511 \mathrm{keV}$, whereas ${ }^{7} \mathrm{Be}$ and ${ }^{22} \mathrm{Na}$ (Gómez-Gomar et al. 1998, [34]), which decay much later (when the envelope is optically thin), are the sources that power line emission at 478 and $1275 \mathrm{keV}$, respectively. ${ }^{26} \mathrm{Al}$ is another important radioactive isotope that can be synthesized during nova outbursts, although only its cumulative emission can be observed because of its slow decay. We will briefly focus on the corresponding nuclear paths leading to the synthesis of the above-mentioned gamma-ray emitters, with special emphasis on the nuclear uncertainties associated with the relevant reaction rates.

\subsection{1 ${ }^{7} \mathrm{Be}-{ }^{7} \mathrm{Li}$}

${ }^{3} \mathrm{He}(\alpha, \gamma){ }^{7} \mathrm{Be}$ is the main reaction leading to ${ }^{7} \mathrm{Be}$ synthesis, which is transformed into ${ }^{7} \mathrm{Li}$ by means of an electron capture, emitting a $\gamma$-ray photon of $478 \mathrm{keV}$. The contribution of nova outbursts to the Galactic ${ }^{7} \mathrm{Li}$ content has been regarded as very controversial. Hints from the first pioneering estimate, in the framework of a simple parametric model (Arnould and Nørgaard, 1975, [35]), were confirmed by early hydrodynamic simulations (Starrfield et al., 1978, [36]), assuming however an envelope in-place, thus neglecting the impact of the accretion phase on the evolution. These results were preliminary refuted later on, in terms of parametric one/two zone models (Boffin et al., 1993, [37]), pointing out the critical role played by the ${ }^{8} \mathrm{~B}(\mathrm{p}, \gamma)$ reaction, not included in all previous works (i.e., Arnould and Nørgaard, 1975, [35], Starrfield et al., $1978,[36])$, and claiming therefore for an unlikely synthesis of ${ }^{7} \mathrm{Li}$ in novae. The scenario was later revisited by Hernanz et al. (1996) [38] and José and Hernanz (1998) [14], who performed new hydrodynamic calculations, taking into account both the accretion and explosion stages, and a full reaction network (including ${ }^{8} \mathrm{~B}(\mathrm{p}, \gamma)$ ). These studies confirmed that the Be-transport mechanism (Cameron, $1955,[39])$ is capable of producing large amounts of ${ }^{7} \mathrm{Li}$ during nova explosions.

Among the key issues that may affect ${ }^{7} \mathrm{Li}$ synthesis in novae, one of the most critical ones is the final amount of ${ }^{3} \mathrm{He}$ that survives the early rise in temperature when the thermonuclear runaway ensues. In particular, the different timescales to reach $T_{\text {peak }}$ achieved for $\mathrm{CO}$ and $\mathrm{ONe}$ novae, which deeply depend on the initial ${ }^{12} \mathrm{C}$ content in the envelope, lead to larger amounts of ${ }^{7} \mathrm{Be}$ in $\mathrm{CO}$ novae (which survives destruction through $(\mathrm{p}, \gamma)$ reactions because of the very efficient inverse photodisintegration reaction on $\left.{ }^{8} \mathrm{~B}\right)$. No relevant nuclear uncertainties in the domain of nova temperatures affect the main reaction rates for ${ }^{7} \mathrm{Li}$ synthesis. It is also worth noting that the potential contribution of classical novae to the Galactic ${ }^{7} \mathrm{Li}$ content is rather small (less than 15\%, according to models). However, a nova contribution is required to match the ${ }^{7} \mathrm{Li}$ content in realistic calculations of Galactic chemical evolution (Romano et al., 1999, [40]; Alibés et al., 2002, [41]).

The observation of ${ }^{7} \mathrm{Li}$ in the ejecta accompanying nova outbursts is extraordinarily challenging. Recently, the presence of this elusive isotope in an ejected nova envelope has been claimed for the first time: an observed feature compatible with the doublet at $6708 \AA$ of Li I has been reported in the spectra of V382 Vel (Nova Velorum 1999; see Della Valle et al., 2002, [42]). However, it has been argued (Shore et al., 2003, [43]) that the observed feature may correspond instead to another low-ionization emission centered at around $6705 \AA$, likely the doublet associated with N I. Attempts to detect the $478 \mathrm{keV}$ line associated to ${ }^{7} \mathrm{Be}-{ }^{7} \mathrm{Li}$ in a classical nova outburst (with the GRS instrument on-board the SMM satellite (Harris et al., 1991, [44]) or with the TGRS spectrometer on-board WIND (Harris et al., 2001, [45])), have also been unsuccessful to date. Unfortunately, current prospects with ESA's INTEGRAL satellite are not much better, because of the small fluxes expected from novae and the limited sensitivity of the INTEGRAL detectors. The predicted detectability distance of the $478 \mathrm{keV}$ line (a $\gamma$-ray signal that may last for about 2 months) is of the order of only $\leq 0.2 \mathrm{kpc}$ for INTEGRAL/SPI (Hernanz and José, 2004, [46]). Future instruments, like the $\gamma$-ray lens MAX, will be better suited to attempt this challenging detection (see Hernanz and José, 2004, [47]).

\section{$2.4 .2^{22} \mathrm{Na}$}

The potential role of ${ }^{22} \mathrm{Na}$ for diagnosis of nova outbursts was first suggested by Clayton and Hoyle (1974) [30]. It decays to a short-lived excited state of ${ }^{22} \mathrm{Ne}$ (with a lifetime of $\tau=3.75 \mathrm{yr}$ ), which de-excites to its ground state by emitting a $\gamma$-ray photon of $1.275 \mathrm{MeV}$. Through this mechanism, nearby ONe novae may provide detectable $\gamma$-ray fluxes. Several experimental verifications of this $\gamma$ ray emission at $1.275 \mathrm{MeV}$ from nearby novae have been attempted in the last twenty years, using balloon-borne experiments and detectors on-board satellites such as HEAO-3, SMM, or CGRO, from which upper limits on the ejected ${ }^{22} \mathrm{Na}$ have been derived. In particular, the observations performed with the COMPTEL experiment onboard CGRO of five recent Ne-type novae as well as observations of standard CO novae (Iyudin et al., 1995, [48]), have led to an upper limit of $3.7 \times 10^{8} \mathrm{M}_{\odot}$ for the ${ }^{22} \mathrm{Na}$ mass ejected by any nova in the Galactic disk. A limit that poses some constraints on pre-existing theoretical models of classical nova explosions.

Synthesis of ${ }^{22} \mathrm{Na}$ in novae proceeds through different reaction paths. In the ${ }^{20} \mathrm{Ne}$-enriched envelopes of $\mathrm{ONe}$ novae (José et al., 1999, [49]), it takes place through ${ }^{20} \mathrm{Ne}(\mathrm{p}, \gamma){ }^{21} \mathrm{Na}$, followed either by another proton capture and a $\beta^{+}$-decay into ${ }^{22} \mathrm{Na}$ (cf., $\left.{ }^{21} \mathrm{Na}(\mathrm{p}, \gamma){ }^{22} \mathrm{Mg}\left(\beta^{+}\right)^{22} \mathrm{Na}\right)$, 
or first decaying into ${ }^{21} \mathrm{Ne}$ before another proton capture ensues (i.e., $\left.{ }^{21} \mathrm{Na}\left(\beta^{+}\right)^{21} \mathrm{Ne}(\mathrm{p}, \gamma){ }^{22} \mathrm{Na}\right)$. Other potential channels, such as proton captures on the seed nucleus ${ }^{23} \mathrm{Na}$, play only a marginal role on ${ }^{22} \mathrm{Na}$ synthesis because of the much higher initial ${ }^{20} \mathrm{Ne}$ content in such ONe models. As for the main destruction channel at nova temperatures, ${ }^{22} \mathrm{Na}(\mathrm{p}, \gamma){ }^{23} \mathrm{Mg}$ competes favorably with ${ }^{22} \mathrm{Na}\left(\beta^{+}\right)^{22} \mathrm{Ne}$. Until very recently, important nuclear uncertainties affected the rates for ${ }^{21} \mathrm{Na}(\mathrm{p}, \gamma)^{22} \mathrm{Mg}$ and ${ }^{22} \mathrm{Na}(\mathrm{p}, \gamma)^{23} \mathrm{Mg}$ (José et al., 1999, [49]), which translated into an uncertainty in the estimated ${ }^{22} \mathrm{Na}$ yields (and ultimately on the maximum detectability distance of the associated $1275 \mathrm{keV}$ line). These uncertainties have been recently reduced by the first direct measurement of the ${ }^{21} \mathrm{Na}(\mathrm{p}, \gamma){ }^{22} \mathrm{Mg}$ rate with the DRAGON recoil separator facility at TRIUMF (Bishop et al., 2003, [50]; D'Auria et al., 2004, [51]; see also the indirect approach by Davids et al., 2003, [52]), and by improving the ${ }^{22} \mathrm{Na}(\mathrm{p}, \gamma)^{23} \mathrm{Mg}$ rate through spectroscopies studies of the ${ }^{23} \mathrm{Mg}$ nucleus (populated by the reaction ${ }^{12} \mathrm{C}\left({ }^{12} \mathrm{C}, \mathrm{n}\right){ }^{23} \mathrm{Mg}$ ), carried out with Gammasphere at the Argonne National Laboratory (Jenkins et al., 2004, [53]).

\section{$2.4 .3{ }^{26} \mathrm{Al}$}

${ }^{26} \mathrm{Al}$ was discovered in the interstellar medium by the HEAO-3 satellite, through the detection of the $1.809 \mathrm{MeV}$ $\gamma$-ray line (Mahoney et al., 1982, [54]; 1984 [55]), a proof of active Galactic nucleosynthesis. This $\gamma$-ray feature is produced by the $\beta^{+}$-decay $\left(\tau=1.04 \mathrm{Myr}\right.$ ) of ${ }^{26} \mathrm{Al}^{g}$ (ground state) to the first excited state of ${ }^{26} \mathrm{Mg}$, which de-excites to its ground state by emitting a $1.809 \mathrm{MeV}$ photon. The synthesis of ${ }^{26} \mathrm{Al}$ requires moderate peak temperatures, of the order of $2 \times 10^{8} \mathrm{~K}$, and a fast decline (Ward and Fowler, 1980, [56]), conditions that are achieved in typical nova outbursts. A detailed analysis of the way ${ }^{26} \mathrm{Al}$ synthesis proceeds, in the framework of $1 \mathrm{D}$ hydrodynamic calculations (José et al., 1997, [57]; 1999 [49]) reveals the existence of several "seed" nuclei: in particular, ${ }^{24,25} \mathrm{Mg}$ and to some extent ${ }^{23} \mathrm{Na}$ and ${ }^{20,22} \mathrm{Ne}$. The main nuclear reaction path leading to ${ }^{26} \mathrm{Al}$ synthesis is ${ }^{24} \mathrm{Mg}(\mathrm{p}, \alpha){ }^{25} \mathrm{Al}\left(\beta^{+}\right){ }^{25} \mathrm{Mg}(\mathrm{p}, \alpha){ }^{26} \mathrm{Al}^{g}$, whereas destruction is dominated by ${ }^{26} \mathrm{Al}^{g}(\mathrm{p}, \gamma){ }^{27} \mathrm{Si}$.

A question raised during the Nuclear Physics in Astrophysics II Workshop concerns the importance of the uncertainties associated with ${ }^{25} \mathrm{Mg}(\mathrm{p}, \gamma)$ as compared with those significantly affecting the ${ }^{25} \mathrm{Al}(\mathrm{p}, \gamma)$ rate (Coc et al., 1995, [58]; José et al., 1999, [49]; see also Rowland et al., 2004, [59] for a recent update of the ${ }^{23} \mathrm{Na}+\mathrm{p}$ rates and their impact on ${ }^{26} \mathrm{Al}$ production in novae): according to a recent sensitivity study (Iliadis et al., 2002, [60]) in nova nucleosynthesis, the uncertainty associated with the ${ }^{25} \mathrm{Mg}(\mathrm{p}, \gamma)$ rates in the domain of nova nucleosynthesis $(T=0.1-0.4 \mathrm{GK})$ is rather limited (i.e., a factor $0.6-1.8$ deviation from the nominal rate). Hence, their impact on nova nucleosynthesis is negligible (cf., less than a factor of $\sim 2$ change in the ${ }^{25,26} \mathrm{Mg}$ and ${ }^{26} \mathrm{Al}$ yields. In contrast, the ${ }^{25} \mathrm{Al}(\mathrm{p}, \gamma)$ rate is affecting from a much larger uncertainty (a factor 0.01-100 deviation from the nominal rate). This huge uncertainty translates into an uncertainty in the contribution of novae to the Galactic ${ }^{26} \mathrm{Al}$ content (since this reaction determines the fraction of the nuclear path that proceeds through the isomeric ${ }^{26} \mathrm{Al}^{m}$ instead), an estimate that relies critically on two ingredients: the adopted surface composition for $\mathrm{ONe}$ white dwarfs (i.e., the initial ${ }^{24} \mathrm{Mg}$ abundance), and the specific rates adopted for the reactions ${ }^{26} \mathrm{Al}^{g}(\mathrm{p}, \gamma)$ and ${ }^{27} \mathrm{Al}(\mathrm{p}, \alpha)$; see José et al., 1997, [57]). Whereas calculations performed by the Arizona group (Starrfield et al., 1986, [61]; 1998 [13]; 2000 [62]) assume a composition based on hydrostatic models of C-burning nucleosynthesis (Arnett and Truran, 1969, [7]), highly enriched in ${ }^{24} \mathrm{Mg}$ (with a ratio ${ }^{16} \mathrm{O}:{ }^{20} \mathrm{Ne}:{ }^{24} \mathrm{Mg}$ of $1.5: 2.5: 1$ ), the Barcelona group (José and Hernanz, 1998, [14]; José et al., 1997, [57]; 1999 [49]; 2001 [63]) has adopted more recent estimates from stellar evolution calculations of intermediate-mass stars (Ritossa et al., 1996, [9]), that result in a much smaller ${ }^{24} \mathrm{Mg}$ content (cf., ${ }^{16} \mathrm{O}:{ }^{20} \mathrm{Ne}:{ }^{24} \mathrm{Mg}$ is 10:6:1). Calculations based on this new ONe white dwarf composition, with an updated nuclear reaction network, suggest that the contribution of classical nova outbursts to the Galactic ${ }^{26} \mathrm{Al}$ abundance is small (less than $\sim 15 \%$ ), in agreement with the results derived from the COMPTEL/CGRO map of the $1.809 \mathrm{MeV}$ ${ }^{26} \mathrm{Al}$ emission in the Galaxy (see Diehl et al., 1995, [64]), which points towards young progenitors (type II supernovae and Wolf-Rayet stars).

\section{$2.4 .4^{13} \mathrm{~N}-{ }^{18} \mathrm{~F}$}

The most intense emission predicted in $\gamma$-rays for classical novae is the $511 \mathrm{keV}$ line and a continuum at lower energies with a cut-off due to photoelectric absorption at about $20-630 \mathrm{keV}$. This prompt emission, that lasts only for a few hours after $T_{\text {peak }}$, is caused by the sudden release of positrons from the $\beta^{+}$-decay of the short-lived species ${ }^{13} \mathrm{~N}$ and ${ }^{18} \mathrm{~F}$, that annihilate with the surrounding electrons when the envelope is already transparent to $\gamma$-rays. The detection of this short duration emission is a challenge since this takes place before the nova optical discovery, which rules out any possibility of a pointed observation. Only instruments with a wide field-of-view (e.g., BATSE and TGRS) have a chance to discover serendipitously this prompt emission, provided that they were pointing at the right place at the right time. Several attempts have been carried out to detect these $\gamma$-ray signatures from classical novae, from which only upper limits on the ${ }^{18} \mathrm{~F}$ annihilation line were obtained. Detectability distance estimates of the $511 \mathrm{keV}$ line with the INTEGRAL's spectrometer SPI and of the continuum with the INTEGRAL's imager IBIS are about $4 \mathrm{kpc}$ (for $10 \mathrm{~h}$ of observation; see Hernanz et al., 1999, [33]; Hernanz and José, 2004, [47]). However, the limited field-of-view of these instruments make this detection unlikely. Better chances can be expected with future instruments, such as EXIST (see Hernanz et al., 2002, [65]).

The synthesis of ${ }^{18} \mathrm{~F}(\tau=158 \mathrm{~min})$ is mainly powered by ${ }^{16} \mathrm{O}(\mathrm{p}, \gamma){ }^{17} \mathrm{~F}$, which is followed either by 
${ }^{17} \mathrm{~F}(\mathrm{p}, \gamma){ }^{18} \mathrm{Ne}\left(\beta^{+}\right){ }^{18} \mathrm{~F}$ or by ${ }^{17} \mathrm{~F}\left(\beta^{+}\right){ }^{17} \mathrm{O}(\mathrm{p}, \gamma){ }^{18} \mathrm{~F}$. The dominant destruction channel is ${ }^{18} \mathrm{~F}(\mathrm{p}, \alpha){ }^{15} \mathrm{O}$ plus a minor contribution from ${ }^{18} \mathrm{~F}(\mathrm{p}, \gamma){ }^{19} \mathrm{Ne}$. The effect of the nuclear uncertainties associated with some of these rates (mainly ${ }^{18} \mathrm{~F}(\mathrm{p}, \alpha){ }^{15} \mathrm{O}$, but also ${ }^{18} \mathrm{~F}(\mathrm{p}, \gamma){ }^{19} \mathrm{Ne},{ }^{17} \mathrm{O}(\mathrm{p}, \alpha){ }^{14} \mathrm{~N}$, and ${ }^{17} \mathrm{O}(\mathrm{p}, \gamma){ }^{18} \mathrm{~F}$. See discussion in Hernanz et al., 1999, [33], and Coc et al., 2000, [66]) translates into a significant uncertainty in the expected ${ }^{18} \mathrm{~F}$ yields and, hence, in the corresponding $\gamma$-ray fluxes and maximum detectability distances. The situation has improved recently in which concerns the ${ }^{17} \mathrm{O}+\mathrm{p}$ rates (see Fox et al., 2004, [67], and Chafa et al., 2005, [68]). Further advances to reduce the remaining uncertainties have been achieved through several nuclear physics experiments performed in Oak Ridge (see Bardayan, these proceedings, and references therein) and Louvain-la-Neuve (de Séréville et al. 2003 [69]).

\subsection{The endpoint of nova nucleosynthesis}

Theoretical estimates of the expected endpoint for novae nucleosynthesis suggest that nuclear activity stops around the mass region $A \sim 40$ (cf., calcium), in agreement with the abundance patterns inferred from observations of the ejecta. Indeed, spectroscopic abundance determinations of nova shells include silicon (Nova Aql 1982, QU Vul 1984), sulfur (Nova Aql 1982), chlorine (Nova GQ Mus 1983), argon and calcium (Nova GQ Mus 1983, Nova V2214 Oph 1988, Nova V977 Sco 1989 and Nova V443 Sct 1989), whereas no indication of a significant overproduction with respect to solar abundances has been ever reported for elements above $\mathrm{Ca}$. It is worth mentioning that this conclusion relies on the fact that the temperatures attained in the envelope during the explosion are limited to $T_{\text {peak }}<4 \times 10^{8} \mathrm{~K}$.

The nuclear activity in the $\mathrm{Si}-\mathrm{Ca}$ region has been scarcely analyzed in detail in the context of classical nova outbursts (see Starrfield et al., 1998, [13]; Iliadis et al., 1999, [70]; Wanajo et al., 1999, [71], and José et al., 2001, [63]). It is powered by a leakage from the $\mathrm{NeNa}-\mathrm{MgAl}$ region, where the activity is confined during the early stages of the explosion (see Rowland et al., 2004, [59] for a recent paper on the lack of a closed $\mathrm{NeNa}$-cycle in nova conditions). The main reaction that drives the nuclear activity towards heavier species (i.e., beyond $\mathrm{S}$ ) is mainly ${ }^{30} \mathrm{P}(\mathrm{p}, \gamma)^{31} \mathrm{~S}$, either followed by ${ }^{31} \mathrm{~S}(\mathrm{p}, \gamma){ }^{32} \mathrm{Cl}\left(\beta^{+}\right){ }^{32} \mathrm{~S}$, or by ${ }^{31} \mathrm{~S}\left(\beta^{+}\right){ }^{31} \mathrm{P}(\mathrm{p}, \gamma){ }^{32} \mathrm{~S}$ (José et al., 2001, [63]). The current ${ }^{30} \mathrm{P}(\mathrm{p}, \gamma){ }^{31} \mathrm{~S}$ rate is based on Hauser-Feshbach estimates, which can be rather uncertain at the domain of nova temperatures. To test the effect of this uncertainty on the predicted yields, a series of hydrodynamic calculations has been performed (José et al., 2001, [63]), modifying arbitrarily the nominal rate. Hence, for a high ${ }^{30} \mathrm{P}(\mathrm{p}, \gamma){ }^{31} \mathrm{~S}$ rate (i.e., 100 times the nominal one), the final ${ }^{30} \mathrm{Si}$ yields are dramatically reduced by a factor of 30 , whereas for a low ${ }^{30} \mathrm{P}(\mathrm{p}, \gamma){ }^{31} \mathrm{~S}$ rate $($ i.e., 0.01 times the nominal one), the final ${ }^{30} \mathrm{Si}$ yields are slightly increased by a factor of 5 , whereas isotopes above silicon are reduced by a factor of $\sim 10$, with a dramatic impact on estimates of the composition of the ejecta and of the chemical pattern of meteoritic grains to be condensed in the ejected shells. Attempts to reduce the uncertainty associated with this rate are currently in progress at several nuclear physics facilities, including ORNL (Oak Ridge), ANL (Argonne), and JYFL (University of Jyväskylä). In particular, a ${ }^{12} \mathrm{C}\left({ }^{20} \mathrm{Ne}, \mathrm{n}\right){ }^{31} \mathrm{~S}$ experiment to study protonunbound levels in ${ }^{31} \mathrm{~S}$ has been performed in ANL with gammasphere, with the goal to determine their corresponding spins and parities (see Jenkins et al., 2005, [72]).

\section{X-ray bursts}

Accretion onto neutron stars in close binary systems can also give rise to some nova-like events known as X-ray bursts. Because of the stronger surface gravity in a neutron star (as compared with that of a white dwarf), temperatures and densities in the accreted envelope during X-ray bursts are typically an order of magnitude greater than in a typical nova outburst (see pioneering models by Woosley and Taam, 1976, [73]; Joss, 1977, [74], and Maraschi and Cavaliere, 1977, [75]). As a result, detailed nucleosynthesis studies involve several hundreds of isotopes (up to the so-called SnSbTe cycle. See Schatz et al., 2001, [76]) and thousands of nuclear reactions. Indeed, the main reaction flow moves far away from the valley of stability, and even merges with the proton drip line beyond $A=38$ (Schatz et al., 1999, [77]). Until recently, because of computational limitations, many simulations of X-ray bursts were performed in the framework of reduced nuclear reaction networks. Examples include network endpoints around Ni (Woosley and Wallace, 1984, [78]; Taam, 1993, [79]; Taam, 1996, [80]), Ga (José and Moreno, 2003, [81]), Se (Hanawa et al., 1983, [82]), Kr (Koike et al., 1999, [83]), or Y (Wallace and Woosley, 1981, [84]). Indeed, Wallace and Woosley (1984) [85] reached ${ }^{96} \mathrm{Cd}$, but in the context of a reduced 16-nuclei network. On the other hand, Schatz et al. (1999) [77], (2001) [76] have carried out very detailed nucleosynthesis calculations, with a complete reaction network up to the SnSbTe mass region, but using a simple one-zone approach. Similar criticism can be applied to other works, such as Moreno et al. (2001) [86], that reached $\mathrm{Pd}$, and Koike et al. (2004) [87], whose network extended up to Bi. Recent hydrodynamic simulations with a reasonably extended network containing 298 isotopes (up to $\mathrm{Te}$ ) have been performed by Fisker et al. (2004) [88]. An unprecedented attempt, coupling detailed hydrodynamic stellar models in one-dimension with a complete nuclear reaction network (up to 1300 isotopes, in the framework of an adaptive network) has been recently performed by Woosley et al. (2004) [89].

\subsection{Nucleosynthesis in X-ray bursts}

Contrary to classical novae, where the main nuclear activity is driven by proton-capture reactions in competition with $\beta^{+}$-decays, X-ray bursts are triggered by a combination of nuclear reactions, including H-burning (via rpprocess) and He-burning (that initiates with the triple 
$\alpha$-reaction, and is followed both by the breakout of the CNO cycle by ${ }^{14,15} \mathrm{O}+\alpha$, plus a competition of proton captures and $(\alpha, \mathrm{p})$ reactions - the so-called $\alpha$ p-process). Initially, the CNO breakout is led by ${ }^{15} \mathrm{O}(\alpha, \gamma){ }^{19} \mathrm{Ne}$ (see Fisker et al., 2005, [90] for a recent study of the impact of the ${ }^{15} \mathrm{O}(\alpha, \gamma)$ rate on the bursting behavior of an accreting neutron star), which is followed by two consecutive proton-captures through ${ }^{20} \mathrm{Na}$ and ${ }^{21} \mathrm{Mg}$, where the flow faces strong photodisintegration reactions on ${ }^{22} \mathrm{Al}$. Following ${ }^{21} \mathrm{Mg}$-decay, they flow shifts through ${ }^{21} \mathrm{Na}(\mathrm{p}, \gamma){ }^{22} \mathrm{Mg}$ (see D'Auria et al., 2004, [51] for a recent update of this rate for X-ray bursts conditions). As the temperature rises, and enough ${ }^{14} \mathrm{O}$ is built up through the triple- $\alpha$ reaction, followed by ${ }^{12} \mathrm{C}(\mathrm{p}, \gamma){ }^{13} \mathrm{~N}(\mathrm{p}, \gamma){ }^{14} \mathrm{O}$, an alternative path through ${ }^{14} \mathrm{O}(\alpha, \mathrm{p}){ }^{17} \mathrm{~F}$ reaction dominates the flow (see Champagne and Wiescher, 1992, [91], and Woosley et al., 2004, [89]), by-passing the ${ }^{15} \mathrm{O}(\alpha, \gamma){ }^{19} \mathrm{Ne}$ link to ${ }^{21} \mathrm{Na}$ through ${ }^{17} \mathrm{~F}(\mathrm{p}, \gamma){ }^{18} \mathrm{Ne}(\alpha, \mathrm{p}){ }^{21} \mathrm{Na}$, where ${ }^{18} \mathrm{Ne}(\alpha, \mathrm{p}){ }^{21} \mathrm{Na}$ represents the main path towards heavier species. The rates for these breakout reactions have huge associated uncertainties (see Görres et al., 1995, [92], and Mao et al., 1996, [93]) and are subject to intense experimental studies both at stable and radioactive beam facilities. Of special interest is the fact that $\mathrm{H}$-burning continues after the peak of the explosion through the rp-process, which extends the main path much beyond ${ }^{56} \mathrm{Ni}$, up to the SnSbTe region (see Fisker et al., 2005, [90] for a detailed account of the main nuclear paths at different stages of the TNR).

A major drawback in the modeling of X-ray bursts comes from the lack of observational nucleosynthetic constraints (beyond obvious implications for the physics of the neutron star crusts). This results from the difficulty of ejection in the strong gravitational well of a neutron star. A recent attempt to overcome this limitation has been provided by high-resolution spectra obtained with XMM-Newton (Cottam et al., 2002, [94]) that bring an insight into the chemical species present in the envelope at different epochs (determinations include $\mathrm{C}, \mathrm{N}, \mathrm{O}, \mathrm{Ne}$, or Fe, with different degrees of ionization; see also Chang et al., 2005, [95] for a very recent study on formation of resonant atomic lines during X-ray bursts).

It is important to stress that, contrary to nova outbursts, convection is not that critical in the progress of the TNR for X-ray bursts. This can be tested by artificially turning-off convective transport in a series of numerical tests: whereas convection is the key ingredient to power the expansion and ejection stages in a nova outburst (through the transport of the short-lived species ${ }^{13} \mathrm{~N},{ }^{14,15} \mathrm{O}$ and ${ }^{17} \mathrm{~F}$ ), an X-ray burst is not deeply affected by the lack of convection. Indeed, recent X-ray burst models (Woosley et al., 2004, [89]) point out that convection sets in during a very short time (of the order of a few seconds).

\subsection{Future challenges: superbursts}

Whereas regular, type I X-ray bursts exhibit some common features in terms of duration, energetics, and recurrence times, a few extremely energetic events have been recently reported thanks to better performances in monitoring achieved with X-ray satellites (i.e., BeppoSAX, Chandra, or XMM-Newton). The first observation of a superburst was reported by Cornelisse et al. (2000) [96] in the context of a "common" type I bursting source (cf., the BeppoSAX source $4 \mathrm{U} 1735$ - 44). Several other sources have been identified since then, including 4U 1636 - 53 for which two superbursts have been reported already.

According to their distinctive characteristics, superbursts represent some sort of extreme X-ray bursts: they have long duration, with a typical (exponential) decay time ranging from 1 to 3 hours (including an extreme case, KS 1731 - 260, which lasted for more than 10 hours. See Kuulkers et al., 2002, [97] for details), extremely energetic (about $\sim 1000$ times more than a typical X-ray burst), and with much longer recurrence periods $(4.7 \mathrm{yr}$ for the system $4 \mathrm{U} 1636$ - 53, the only one in which two superbursts have been observed to date. See Wijnands, 2001, [98]). Indeed, the durations and energetics of superbursts suggest that they result from thermonuclear flashes occurring in fuel layers at much greater depth than for typical Xray bursts, more likely, in the C-rich ashes resulting from type I X-ray bursts (Cumming and Bildsten, 2001, [99]. See also Woosley and Taam, 1976, [73]; Taam and Picklum, 1978, [100], and Brown and Bildsten, 1998, [101]). Controversy remains as how much carbon is left after a type I burst: Schatz et al. (1999) [77], (2001) [76] have indeed shown than most of the $\mathrm{C}$ is burnt during the previous $\mathrm{H} / \mathrm{He}$ burning. However, Cumming and Bildsten (2001) [99] concluded that even small amounts of carbon are enough to power a superburst (especially in neutron star oceans enriched from the heavy ashes driven by the rp-process). More theoretical efforts to test these ideas are required to shed light into this question.

\section{Outlook}

A final comment on nuclear physics input requirements as well as on reaction rates that need to be improved to overcome their huge associated uncertainties: the situation concerning nova modeling is quite well defined. Significant experimental information is already available and we are confident that, in the near future, novae will become the first explosive stellar site for which most (if not all) of the relevant nuclear physics input will be primarily based on experimental information. Main uncertainties are localized in only a handful of nuclear reaction rates (namely, ${ }^{18} \mathrm{~F}(\mathrm{p}, \alpha),{ }^{25} \mathrm{Al}(\mathrm{p}, \gamma)$ and ${ }^{30} \mathrm{P}(\mathrm{p}, \gamma)$ ), for which several experiments have been already proposed in different facilities.

In contrast, the situation is much more complex as regards X-ray bursts: nuclear physics requirements include mass measurements along the rp-process path (see Clark et al., 2004, [102] for a recent mass determination for ${ }^{68} \mathrm{Se}$ ), and a much better knowledge of key reactions and effective lifetimes at the waiting points (in particular, ${ }^{30} \mathrm{~S}(\alpha, \mathrm{p})$ and ${ }^{34} \operatorname{Ar}(\alpha, p)$; see Fisker et al., 2004, [88]) under rp-process conditions. However, it is important to stress that, far from the waiting points, the situation is not well settled. 
Indeed, recent hydrodynamic calculations (see details in D'Auria et al., 2004, [51]) suggest that single nuclear reactions (in particular, ${ }^{21} \mathrm{Na}(\mathrm{p}, \gamma)$ ) are not so important, since the much higher temperatures achieved in X-ray bursts allow alternative paths for the main nuclear flows. The future looks bright for X-ray bursts physics, since future facilities like RIA in the U.S., or FAIR at GSI (Germany) have already identified the mass region of interest for these stellar explosions as part of their future research programs.

\section{References}

1. J. Grindlay et al., Astrophys. J. 205, L127 (1976).

2. R.D. Belian et al., Astrophys. J. 206, L135 (1976).

3. J.H. Swank et al., Mon. Not. R. Astron. Soc. 182, 349 (1978)

4. A.W. Shafter, in Classical Nova Explosions, edited by M. Hernanz, J. José, AIP Conf. Proc. 637, 462 (2002).

5. B. Warner, in Cataclysmic Variable Stars (Cambridge University Press, Cambridge, 1995).

6. T. Strohmayer, L. Bildsten, in Compact Stellar X-Ray Sources, edited by W.H.G. Lewin, M. van der Klis (Cambridge University Press, 2006) in press.

7. W.D. Arnett, J.W. Truran, Astrophys. J. 157, 339 (1969).

8. I. Domínguez et al., Astrophys. J. 419, 268 (1993).

9. C. Ritossa et al., Astrophys. J. 460, 489 (1996).

10. J. José et al., Astrophys. J. 597, L41 (2003).

11. B. Davids et al., Phys. Rev. C 67, 065808 (2003).

12. J. José et al., Nucl. Phys. A, Special Volume, in press (2006).

13. S. Starrfield et al., Mon. Not. R. Astron. Soc. 296, 502 (1998).

14. J. José, M. Hernanz, Astrophys. J. 494, 680 (1998).

15. A. Kovetz, D. Prialnik, Astrophys. J. 477, 356 (1997).

16. O. Yaron et al., Astrophys. J. 623, 398 (2005).

17. S. Starrfield, in Classical Nova Explosions, edited by M. Hernanz, J. José, AIP Conf. Proc. 637, 89 (2002).

18. R.D. Gehrz et al., Publ. Astron. Soc. Pac. 110, 3 (1998).

19. A. Evans, in The Physics of Classical Novae, edited by A. Cassatella, R. Viotti (Springer-Verlag, Berlin, 1990) p. 253.

20. J.M.C. Rawlings, A. Evans, in Classical Nova Explosions, edited by M. Hernanz, J. José, AIP Conf. Proc. 637, 270 (2002).

21. R.D. Gehrz, in Classical Nova Explosions, edited by M. Hernanz, J. José, AIP Conf. Proc. 637, 198 (2002).

22. D.D. Clayton, F. Hoyle, Astrophys. J. 203, 490 (1976).

23. S. Amari et al., Astrophys. J. 551, 1065 (2001).

24. S. Amari, New Astron. Rev. 46, 519 (2002).

25. S. Starrfield et al., in Astrophysical Implications of the Laboratory Study of Presolar Materials, edited by T. Bernatowicz, E. Zinner, AIP Conf. Proc. 402, 203 (1997).

26. J. José et al., Publ. Astron. Soc. Aust. 20, 351 (2003).

27. J. José et al., Astrophys. J. 612, 414 (2004).

28. S.N. Shore, R.D. Gehrz, Astron. Astrophys. 417, 695 (2004).

29. S. Starrfield et al., Astrophys. J. 176, 169 (1972).

30. D.D. Clayton, F. Hoyle, Astrophys. J. 187, L101 (1974).

31. D.D. Clayton, Astrophys. J. 244, L97 (1981).

32. M.D. Leising, D.D. Clayton, Astrophys. J. 323, 159 (1987).
33. M. Hernanz et al., Astrophys. J. 526, L97 (1999).

34. J. Gómez-Gomar et al., Mon. Not. R. Astron. Soc. 296, 913 (1998).

35. M. Arnould, H. Nørgaard, Astron. Astrophys. 42, 55 (1975).

36. S. Starrfield et al., Astrophys. J. 222, 600 (1978).

37. H.M.J. Boffin et al., Astron. Astrophys. 279, 173 (1993).

38. M. Hernanz et al., Astrophys. J. 465, L27 (1996).

39. A.G.W. Cameron, Astrophys. J. 121, 144 (1955).

40. D. Romano et al., Astron. Astrophys. 352, 117 (1999).

41. A. Alibés et al., Astrophys. J. 571, 336 (2002).

42. M. Della Valle et al., Astron. Astrophys. 390, 155 (2002).

43. S.N. Shore et al., Astrophys. J. 125, 1507 (2003).

44. M.J. Harris et al., Astrophys. J. 375, 216 (1991).

45. M.J. Harris et al., Astrophys. J. 563, 950 (2001).

46. M. Hernanz, J. José, in Proceedings of the V INTEGRAL Workshop, ESA SP-552, edited by U. Schönfelder, G. Lichti, C. Winkler (ESA Publ. Division: ESTEC, Noordwijk, 2004) p. 95.

47. M. Hernanz, J. José, New Astron. Rev. 48, 35 (2004).

48. A.F. Iyudin et al., Astron. Astrophys. 300, 422 (1995).

49. J. José et al., Astrophys. J. 520, 347 (1999).

50. S. Bishop et al., Phys. Rev. Lett. 90, 162501 (2003).

51. J.M. D'Auria et al., Phys. Rev. C 69, 065803 (2004).

52. B. Davids et al., Phys. Rev. C 68, 055805 (2003).

53. D. Jenkins et al., Phys. Rev. Lett. 92, 031101 (2004).

54. W.A. Mahoney et al., Astrophys. J. 262, 742 (1982).

55. W.A. Mahoney et al., Astrophys. J. 286, 578 (1984).

56. R.A. Ward, W.A. Fowler, Astrophys. J. 238, 266 (1980).

57. J. José et al., Astrophys. J. 479, L55 (1997).

58. A. Coc et al., Astron. Astrophys. 299, 479 (1995).

59. C. Rowland et al., Astrophys. J. 615, L37 (2004).

60. C. Iliadis et al., ApJS 142, 105 (2002).

61. S. Starrfield et al., Astrophys. J. 303, L5 (1986).

62. S. Starrfield et al., ApJS 127, 485 (2000).

63. J. José et al., Astrophys. J. 560, 897 (2001).

64. R. Diehl et al., Astron. Astrophys. 298, 445 (1995).

65. M. Hernanz et al., New Astron. Rev. 46, 559 (2002).

66. A. Coc et al., Astron. Astrophys. 357, 561 (2000).

67. C. Fox et al., Phys. Rev. Lett. 93, 081102 (2004).

68. A. Chafa et al., Phys. Rev. Lett. 95, 031101 (2005).

69. N. de Séréville et al., Phys. Rev. C 67, 052801 (2003).

70. C. Iliadis et al., Astrophys. J. 524, 434 (1999).

71. S. Wanajo et al., Astrophys. J. 523, 409 (1999).

72. D. Jenkins et al., Phys. Rev. C 72, 031303 (2005).

73. S.E. Woosley, R.E. Taam, Nature 263, 101 (1976).

74. P.C. Joss, Nature 270, 310 (1976).

75. L. Maraschi, A. Cavaliere, in Highlights in Astronomy, edited by E. Muller, vol. 4, Part I (Reidel, Dordrecht) p. 127.

76. H. Schatz et al., Phys. Rev. Lett. 86, 3471 (2001).

77. H. Schatz et al., Astrophys. J. 524, 1014 (1999).

78. S.E. Woosley, R.K. Wallace, in High Energy Transients in Astrophysics, edited by S.E. Woosley (AIP Press, New York, 1984) p. 273.

79. R.E. Taam et al., Astrophys. J. 413, 324 (1993).

80. R.E. Taam et al., Astrophys. J. 459, 271 (1996).

81. J. José, F. Moreno, Nucl. Phys. A 718, 602 (2003).

82. T. Hanawa et al., Publ. Astron. Soc. Jpn. 35, 491 (1983).

83. O. Koike et al., Astron. Astrophys. 342, 464 (1999).

84. R.K. Wallace, S.E. Woosley, Astrophys. J. Suppl. 45, 389 (1981). 
85. R.K. Wallace, S.E. Woosley, in High Energy Transients in Astrophysics, edited by S.E. Woosley (AIP Press, New York, 1984) p. 319.

86. F. Moreno et al., Nucl. Phys. A 688, 447 (2001).

87. O. Koike et al., Astrophys. J. 603, 242 (2004).

88. J.L. Fisker et al., Astrophys. J. 608, L61 (2004).

89. S.E. Woosley et al., Astrophys. J. Suppl. 151, 75 (2004)

90. J.L. Fisker et al., Nucl. Phys. A 752, 604 (2005).

91. A.E. Champagne, M. Wiescher, Annu. Rev. Nucl. Part. Sci. 42, 39 (1992)

92. J. Görres et al., Phys. Rev. C 51, 392 (1995).
93. Z.Q. Mao et al., Phys. Rev. C 53, 1197 (1996).

94. J. Cottam et al., Nature 420, 51 (2002).

95. P. Chang et al., Astrophys. J. 629, 998 (2005).

96. R. Cornelisse et al., Astron. Astrophys., 357, L21 (2000).

97. E. Kuulkers et al., Astron. Astrophys. 382, 503 (2002).

98. R. Wijnands, Astrophys. J. 554, L59 (2001).

99. A. Cumming, L. Bildsten, Astrophys. J., 559, L127 (2001).

100. R.E. Taam, R. Picklum, Astrophys. J. 224, 210 (1978).

101. E. Brown, L. Bildsten, Astrophys. J., 496, 915 (1998).

102. J.A. Clark et al., Phys. Rev. Lett. 92, 192501 (2004). 